\title{
Fahr's Disease: An Uncommon Cause of Intracranial Calcification in Children
}

\author{
Nayek K', Pati S², Banerjee $A^{3}$, Bhattacharjya $S^{4}$ \\ ${ }^{1}$ Dr. Kaustav Nayek, MBBS, MD, Associate Professor, ${ }^{2}$ Dr. Sananda Pati, MBBS, PG Trainee in Paediatrics, ${ }^{3}$ Dr. Arundhati \\ Banerjee, MBBS, PG Trainee in Paediatrics, ${ }^{4}$ Dr. Sudipta Bahttacharjya, MBBS, PG Trainee in Paediatrics. All from the \\ Department of Paediatrics, Burdwan Medical College, Burdwan, West Bengal, India.
}

Address for correspondence: Dr. Kaustav Nayek, E-mail: kaustav25@yahoo.co.in

\begin{abstract}
Idiopathic bilateral striopallidodentate calcification or Fahr's disease is a degenerative disease normally found in the $5-6^{\text {th }}$ decade of life. Here we report one of the youngest cases of sporadic Fahr's disease presenting at five years of age with minimal clinical features but extensive intracranial calcification, a rarity.
\end{abstract}

\section{Introduction}

diopathic bilateral striopallidodentate calcification or Fahr's disease is a degenerative disease normally found in the $5-6^{\text {th }}$ decade of life with autosomal dominant inheritance ${ }^{1,2,3,4}$. Few sporadic cases are reported. Here we report one of the youngest cases of sporadic Fahr's disease presenting at five years of age with minimal clinical features but extensive intracranial calcification, a rarity.

Fahr's disease was the diagnosis of exclusion ${ }^{1,4,5}$ based on clinical, biochemical, and radiological features, ruling out the more common causes of intracranial calcification in children. Symptomatic management was done and the child is currently on follow up. Extensive intracranial calcification is rare in children and needs proper investigation to find out the cause to guide management.

\section{The Case}

A $5 y$ r old girl reported in our OPD with the chief complains of brief episodes of convulsion involving the whole body for one week. There was no history of associated fever, trauma, abnormal behaviour, weakness, abnormal movement, unusual colour or odour of urine, bleeding manifestation, jaundice. No significant past history. She was born out of a nonconsanguinous marriage with uneventful antenatal, perinatal and postnatal period. She had achieved normal developmental milestones. Her parents and younger sibling were asymptomatic.The history was classical of generalised tonic clonic convulsion of epilepsy.
General survey revealed normal facies and anthropometric parameters. No neurocutaneous markers. Neurological examination showed no meningeal signs or signs of raised intracranial tension. Higher function, speech, cranial nerves were normal. There was no neurodeficit, paraesthesias, extrapyramidal symptoms or gait disturbances. Examination of all other systems was normal.

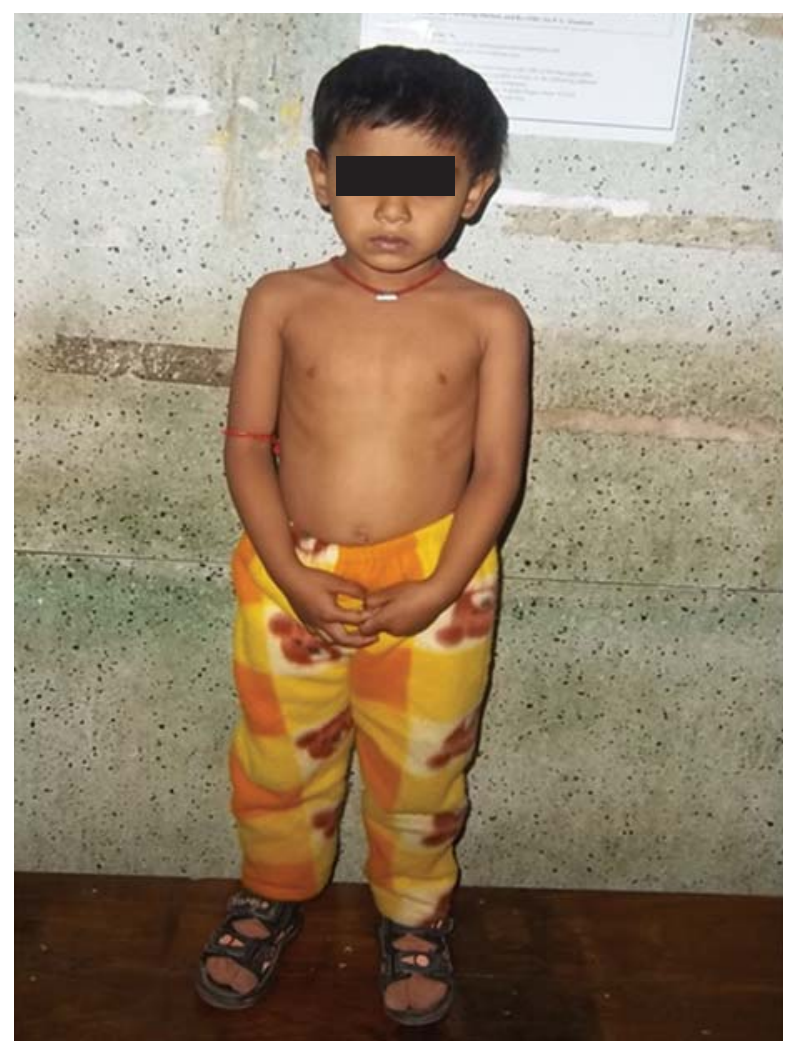

Fig 1: Showing the patient with Fahr's Disease at an early age 


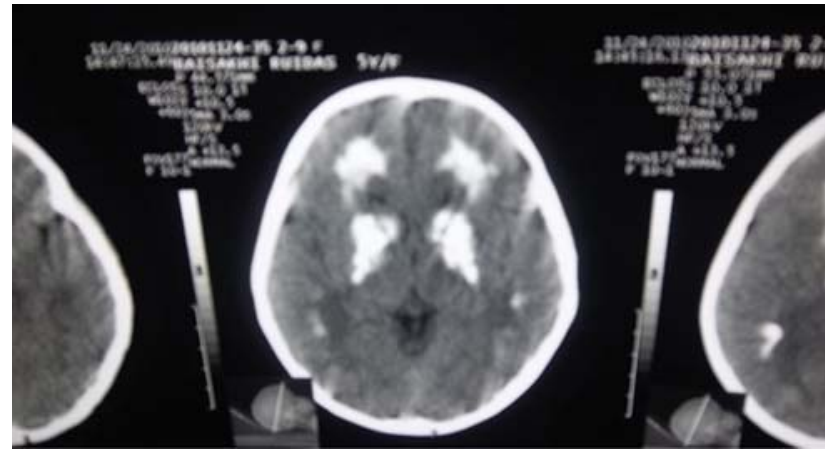

Fig 2: CT Scan (head) of the patient showing Sub cortical white matter and basal ganglia calcification

CECT scan and EEG were ordered in line with the diagnosis of epilepsy. EEG showed suppression burst pattern. CECT showed bilaterally symmetrical non enhancing hyperdense lesions involving subcortical and periventricular white matter, basal ganglia, white matter of cerebellum, consistent with calcification.

Serum calcium, albumin, phosphorus, alkaline phosphataseand parathormonelevelswere normal ruling out hypoparathyroidism, pseudohypoparathyroidism, hyperparathyroidism. History and examination ruled out hypocalcemia or hypercalcemia. CT scan of parents and younger sibling were normal.

The child was diagnosed as a sporadic case of fahr's disease and managed symptomatically with antiepileptics. She's on follow up for the last 6 mnths, seizures have been controlled and there are no new symptoms. The younger sibling is also asked to have regular follow ups.

\section{Discussion}

The important causes of intracranial calcification are metastatic calcification as in hypoparathyroidism, pseudohypoparathyroidism, hyperparathyroidism, dihydropteridine deficiency ${ }^{6}$. Dystrophic calcification seen in following encephalitis, TORCH infection parasitosis, ischaemic or haemorrhagic stroke, A-Vmalformations, tumours, phakomatoses, MELAS and Fahr's disease ${ }^{5,6}$.

There was no history suggestive of stroke or encephalitis. Clinical examination and biochemical parameters ruled out hypo or hypercalcemia, MELAS. Clinical and radiological features were inconsistent with tumours, neurocysticercosis, TORCH infection, A-V malformations, phakomatoses. Thus Fahrs disease was the diagnosis of exclusion ${ }^{1,4,5}$.

Fahr's disease is a rare, genetically dominant, inherited neurological disorder characterized by

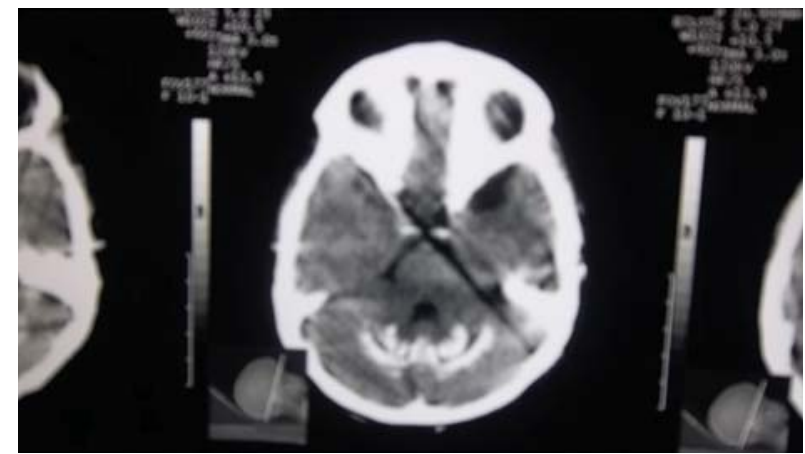

Fig 3: CT Scan of the patient showing Cerebellar Calcification

abnormal deposits of calcium in areas of the brain that control movement, including the basal ganglia and the cerebral cortex. However sporadic ${ }^{1,4}$ cases have been reported, Our case was one such.

The calcium deposits in the brain may occur before the onset of the symptoms, usually in the third decade of life. Although it may also be evident in childhood ${ }^{4,5}$ and with advancing age the amount of calcification increases. In Fahr's disease the mineral deposits tend to be selective for small capillaries and small vessels of white matter, which is different from that in atherosclerosis ${ }^{4}$. The calcification may include endothelial and stromal vascular cells as well as the interstitium. However, the local circulatory disturbances such as regional ischemia have been regarded as the primary event precipitating the deposition of calcium as well as other minerals. Other contributed factors are abnormality in the calcium metabolism or local inflammatory process ${ }^{4,5}$. Also, the calcification could be a primary event occurring without preceding circulatory dysfunction, since a significant familial type suggests either autosomal recessive or dominant inheritance ${ }^{1,4}$. Brain calcification without symptoms such as the small calcifications in the basal ganglia, and less commonly in the dentate nucleus of the cerebellum can occur in elderly patients but is of rare occurrence in children.

Symptoms of the disorder may include deterioration of motor function, dementia, seizures, headache, dysarthria (poorly articulated speech), spasticity (stiffness of the limbs) and spastic paralysis, eye impairments, and athetosis (involuntary, writhing movements) ${ }^{1,4,5}$. Fahr's disease can also include symptoms characteristic of Parkinson's disease such as tremors, muscle rigidity, a mask-like facial appearance, shuffling gait, and a "pill-rolling" motion of the fingers. These symptoms generally occur later in the development of the disease. More common symptoms include dystonia (disordered muscle tone) and chorea (involuntary, rapid, jerky movements). Age of onset is typically in the 40 s or 50 s, although it can occur at any time in childhood or 
adolescence. In our cases it presented with generalised tonic clonic convulsion as early as five years age.

There is no cure for Fahr's Syndrome, nor is there a standard course of treatment. Treatment addresses symptoms on an individual basis. Our case has been effectively treated with sodium valproate and is on follow up.

The prognosis for any individual with Fahr's disease is variable and hard to predict. There is no reliable correlation between age, extent of calcium deposits in the brain, and neurological deficit. Since the appearance of calcification is age-dependent, a CT scan could be negative in a gene carrier who is younger than the age of $55^{2,3}$. Genetic analysis could not be done due to lack of facility for the same in our set up.

Idiopathic Fahr's disease has been linked to IBGC1 locus on chromosome $14 \mathrm{q}$ in some cases ${ }^{2,3}$. However research is needed to understand the actions of the genes involved in this disorder. Finding these could lead to effective ways to treat and prevent Fahr's disease.

\section{References}

1. EBillard C, Dulac O, Boulouche J, Echenne B, Lebon P, Motte J, Robain O, Santini JJ. Encephalopathy with calcifications of the basal ganglia in children: a reappraisal of Fahr's syndrome with respect to 14 new cases. Neuropediatrics 1989;20:12-19.

2. Geschwind DH, Loginov M, Stern JM. Identification of a locus on chromosome $14 \mathrm{q}$ for idiopathic basal ganglia calcification (Fahr disease). Am J Hum Genet 199965:764-72.

3. Brodaty $H$, Mitchell P, Luscombe G, Kwok JBJ, Badenhop RF, McKenzie R, Schofield P R. Familial idiopathic basal ganglia calcification (Fahr's disease) without neurological, cognitive and psychiatric symptoms is not linked to the IBGC1 locus on chromosome 14q. Hum Genet 2002110:8-14.

4. R Malik, VK Pandya, D Naik. FAHR'S Disease - A Rare Neurodegenerative Disorder. Ind J Radiol Imag 2004; 14:383-84.

5. Bala V. Manyam. What is and what is not 'Fahr's disease'. Parkinsonism \& Related Disorders 2005; 11:73-80.

6. Venugopal A, Acharya P, Kishore MS. Rare: Extensive intracranial calcification in idiopathic hypoparathyroidism; presentation in an adolescent. Indian J Radiol Imaging 2006;16:181-2.

\section{How to cite this article?}

Nayek K, Pati S, Banerjee A, Bhattacharjya S. Fahr's Disease: An Uncommon Cause of Intracranial Calcification in Children. J Nepal Paediatr Soc 2012;32(1):76-78. 